\title{
Contínuo pendular de la investigación en la administración internacional de las organizaciones:del positivismo funcionalista a la investigación cualitativa y etnográfica.
}

\section{Pendulum continuum of investigation in international management of organizations: from functionalist positivism to qualitative and ethnographic research}

Jose G. Vargas-Hernandez

\begin{abstract}
Resumen
El objetivo de este trabajo es analizar la construcción teórico metodológico de explicaciones de la realidad de los fenómenos de la administración internacional de las organizaciones. Se parte del supuesto de que las teorías clásicas de la administración internacional de las organizaciones han evolucionado en forma paralela a los paradigmas de la ciencia que prevalece en la misma época. En este trabajo se demuestra que los desarrollos teóricos metodológicos han fluctuado en un continuo pendular que va en un extremo de las concepciones epistemológicas positivistas de la ciencia, pasando por el funcionalismo, el estructuralismo, el institucionalismo, el neoinstitucionalismo, el constructivismo social y la perspectiva crítica interpretativa hasta alcanzar el extremo opuesto con los enfoques de la investigación cualitativa y etnográfica.
\end{abstract}

Palabras clave: Administración internacional, constructivismo social y crítica interpretativa, estructuralismo funcional, institucionalismo, investigación cualitativa y etnográfica, neoinstitucionalismo, positivismo funcional

\begin{abstract}
The aim of this paper is to analyze the theoretical methodological construction of reality explanations of international management phenomena in organizations. It departs from the assumption that classical theories of international management of organizations have evolved in parallel form to the prevalent scientific paradigms of that period. It is demonstrated in this paper that the theoretical methodological developments have fluctuated in a pendulum continuum from positivist epistemological conceptions of science in one extreme, going through the functionalism, structuralism, institutionalism, neoinstitutionalism, social constructivism and critical interpretative to achieve the opposite extreme with the qualitative and ethnographic research approaches
\end{abstract}

Key words: international management, social constructivism and critical interpretative approach, functional structuralism, institutionalism, qualitative and ethnographic research, neoinstitutionalism, functional positivism.

1 Profesor Investigador miembro del Sistema Nacional de Investigadores Departamento de Mercadotecnia y Negocios Internacionales Centro Universitario de Ciencias Económico Administrativas U de G. Periférico Norte 799 Edificio G-306 - Zapopan, Jalisco C.P. 45100; México. E-mail: josevargas@cucea.udg.mx

Artigo submetido em fevereiro 2008 e aceito em março de 2009 


\section{Introducción}

En los últimos años cuando los procesos de globalización económica avanzan aceleradamente, se enfatiza la reflexión epistémico y las aperturas a las nuevas conversaciones y a los nuevos acercamientos a la producción de la teoría en el campo de la administración internacional. Los académicos de la administración intentan codificar sus investigaciones epistemológicas y teóricas metodológicas para seguir los pasos de los más desarrollados paradigmas científicos dentro del mundo multiparadigmático de la teoría organizacional a favor de un consenso y una certeza técnica. La alta calidad de la investigación implica estudios científicos de los fenómenos globales - locales (glocales) con el objetivo de construir teorías que pueden implicar, explicar y predecir los fenómenos específicos y relacionarlos con el contexto social y cultural local.

La construcción teórico metodológica de explicaciones de la realidad de los fenómenos de la administración internacional de las organizaciones ha evolucionado sujeta a los paradigmas de la ciencia que prevalecen en la misma época. La concepción positivista epistemológica de la ciencia fue la plataforma sobre la cual se fundamentan los principios teóricos metodológicos del positivismo funcionalista con su retórica objetivista entrelazado con el funcionalismo ortodoxo. Con la introducción de las variables de poder, autoridad, jerarquías y política en los análisis de las estructuras organizacionales internacionales se enfatiza en la corriente epistemológica centrada en un estructuralismo funcionalista de la administración internacional.

El institucionalismo económico sustentado en las teorías puras de la racionalidad económica, la de la agencia principal y los costos de transacción, entre otras, motivaron los análisis de la administración internacional de las organizaciones en una época en que se expanden las operaciones de las corporaciones multinacionales. El neoinstitucionalismo surge con enfoques históricos, sociológicos y de la elección racional que determinan los procesos de la administración internacional de reforma institucional durante la fase de mayor expansión. Las reacciones a las tendencias institucionales se expresan en los modos del constructivismo, la perspectiva de la crítica interpretativa y el postmodernismo dentro del campo de la administración internacional. Finalmente, el péndulo de la epistemología se carga al otro extremo, al aceptar como científicos los análisis de la administración internacional sustentados en fundamentos teórico metodológicos de la investigación cualitativa y etnográfica.

Las ideas y las teorías llegan a ser populares o impopulares en cierto tiempo no porque sean más a menos verdad sino porque los sistemas de valores que las soportan son activados o suprimidos por los desarrollos institucionales o ecológicos (Hofstede, 1980:323). Identificar las mejores prácticas transferibles de una forma predeterminada y categórica con una sociedad o cultura específica, es una situación problemática desde la perspectiva de las principales teorías de administración internacional.

\section{Positivismo funcionalista}

El dominio continuado del pensamiento positivista y funcionalista en la administración internacional es el problema más fundamental. El conocimiento producido en este campo está firmemente enraizado en el funcionalismo ortodoxo, la ciencia normal con su positivismo epistemológico y su retórica objetivista. Importa quién se involucra en el desarrollo de la teoría de la administración internacional bajo los supuestos del conocimiento objetivo requerido por el positivismo que presupone en punto de vista de los académicos. Tradicionalmente ha habido una contradicción fundamental en la investigación positivista de la administración internacional para atender los temas de especificidad del contexto o de la relatividad cultural que debe funcionar de las premisas que son inimical a las epistemologías positivistas.

La administración internacional es la administración intercultural comparativa, los acercamientos de los sistemas de negocios nacionales, las perspectivas institucionalistas o las intersecciones de todos estos tipos con la más amplia literatura de los negocios internacionales y su enfoque en las corporaciones multinacionales ha permanecido en su mayor parte firmemente enraizada en el positivismo funcionalista tradicional. El valor que se atribuye a los valores occidentales, capitalismo, managerialismo, positivismo, etc. se reafirman 
continuamente y toman una forma sofisticada, y constituyen en efecto el marco de referencia subyacente y por las cuales las cambiantes constelaciones estadísticas se evalúan y se deducen las consecuencias culturales.

Para expandir el pensamiento occidental con más perspectivas culturalmente ricas y para identificar las teorías culturalmente libres de la administración incluso a través de cuestiones acerca de la cultura de las teorías administrativas han estado desde los trabajos de Hofstede (1980a). Hofstede clamó que la cultura es una programación mental instalada en las mentes de las personas como una variable interna que da forma a la conducta desde dentro hacia fuera, por lo que para la práctica organizacional, la teoría administrativa y la psicología, es relevante la cultura nacional.

El positivismo se usa para reforzar el privilegio cultural y es parte de la falta de generalización o universalización no inclusiva. Se revela una voluntad por el diálogo como una cobertura de un esencialismo positivista donde las identidades fueron constituidas en términos polarizados y el individualismo como una cobertura para constituir individuos en términos extremadamente colectivistas. Desde la perspectiva del positivismo y el funcionalismo psicológico-sociológico, un segundo momento emana del supuesto de que la cultura debe ser identificable y al menos persistente en el tiempo si cualquier conocimiento acerca de las diferencias culturales se entiende como regularidades generalizables que pueden ser desarrolladas.

La sustitución metafórica implica cambiar del positivismo y funcionalismo a un acercamiento donde el mundo es visto como creándose y recreándose continuamente, negociado y renegociado, y sujeto a lo que Foucault (1979) denomina la ramificación de la gobernanza, el orden jerárquico y más importante, el control. La metáfora de la distancia, por ejemplo, manifiesta intentos positivistas para extender las condiciones del conocimiento administrativo en los dominios tácitos, complejos y abstractos, tales como la cultura.

Se cuestiona hasta qué punto son tales expectativas de la cultura entidades esenciales o artefactos de las premisas teóricas positivistas. El método positivista cuantitativo es ampliamente usado en las investigaciones de transferencias en administración internacional que trata a las pertenencias nacionales y culturales como aspectos esenciales de las organizaciones, pero carece de las herramientas y las metodologías que le permitan ser capaz de exponer el involucramiento de los procesos de transferencia en las relaciones geopolíticas del poder y en el significado que los actores en el campo atribuyen a las prácticas que transfieren o son forzados a adoptar.

Ya sea que se considere la transferencia de conocimiento como lo mejor (Bartlet, Ghosal and Birkinshaw, 2004) o como reflejo de la identidad del corporativo y su sistema de valores (Kostova, 1999) la mayoría de las teorías de transferencia en administración internacional dentro de las multinacionales asume que la elección de la técnica organizacional para ser transferida refleja los objetivos del corporativo o los entendimientos subjetivos de las necesidades de la firma en términos de eficiencia y ventaja competitiva.

Específicamente la teoría sugiere que cuando un recurso, definido como un activo tangible e intangible que una firma controla es valioso, es decir, útil para explotar las oportunidades y/o neutralizar las amenazas en el ambiente y raros, es decir, no comunes, es posible una ventaja competitiva (Barney and Arikan, 2001). La ideología del libre comercio ha estado firmemente entre las élites profesionales y de los formuladores de políticas desde la emergencia del Reino Unido como poder económico global a mitad del siglo XIX llevando a los economistas y a la prensa de finanzas a responder con seguridad que la teoría de la ventaja competitiva todavía aplica de tal forma que el offshoring presentó otra habilidad para incrementar sus habilidades.

A pesar de las teorías recientes que explican los componentes de la administración de recursos y su importancia, se entiende poco relacionado con muchas de las acciones de los administradores que influencian la ventaja competitiva basada en los recursos o cuando esta ventaja importa más. Reflejándose en la ausencia de consideraciones de la administración de recursos en la literatura del punto de vista basado en los recursos, Barney and Arikan (2001:175) que se necesita más trabajo antes del rango completo de temas de implementación de estrategias no incluidos en los trabajos de Barney (1991) que son integrados con la teoría basada en los recursos de la ventaja competitiva. Sirmon, Gove and Hitt (2008) tratan de examinar los efectos 
independientes de los recursos y su administración en los resultados competitivos, y su teoría y su análisis exploran cuando las accione administrativas más importan, así como las características de los recursos, como la flexibilidad del despliegue que afecta la habilidad de los administradores para abundar y desplegar los recursos.

Para capturar el grano fino y la naturaleza fluida de la administración de recursos, Sirmon, Gove and Hitt (2008) se enfocaron en las rivalidades entre las organizaciones específicas, por ejemplo involucramiento directo o contestado, acercamiento que permite más precisión evitando la agregación de numerosos involucramientos competitivos con rivales múltiples en el tiempo, un atributo importante para la teoría enfocada en los efectos de los recursos en el abundamiento y en el despliegue.

Lo que hasta entonces constituyó una bestia demasiado suave o vaga para la epistemología positivista de la ciencia normal llegó a ser el foco de mayor interés.

\section{Estructural funcionalista}

La metateoría estructural funcionalista o la estructuralista funcional subrayan las recientes teorías de menos estructuras jerárquicas de las multinacionales lo cual contribuye a la inhabilidad de este discurso para analizar los contextos culturales como productos de la co-construcción y contestación relacional. El análisis de las multinacionales dentro de los mercados oligopolísticos paralela el desarrollo de las macroperspectivas críticas sobre el comercio y la inversión, particularmente en la escuela de la dependencia (Evans, 1979) y la teoría de los sistemas mundiales (Wallerstein, 2004) en las cuales las relaciones entre los países industriales y en desarrollo son considerados como fundamentalmente de explotación y condicionados en términos desiguales de comercio.

La teoría de la dependencia de recursos sugiere cómo los líderes usan los mecanismos formales el nombramiento en el consejo para cooptar grupos constituyentes externos que apoyen su firma. Un común denominador de la teoría contemporánea de la estrategia del análisis de las 5 fuerzas de Porter (1985) de las estructuras del mercado al punto de vista basado en los recursos de Barney (1991) y más recientemente en las capacidades dinámicas (Teece, Pisano and Shuen, 1997) es que las firmas solamente derivan arriba de las utilidades normales de las ventajas que son protegidas de imitación.

Los clamores al conocimiento objetivo adornados con el funcionalismo y las ciencias normales son un medio para promover los valores y preocupaciones de los grupos sociales hegemónicos. A pesar de que la originalidad se despliegue como una defensa significa la protección de los campos críticos de cuestionamientos del imperialismo intelectual de la ciencia normal, la noción de inconmensurabilidad protege lo último más que lo primero, permitiendo a los miembros de la principal corriente funcionalista llevarse con los negocios como usuales.

El trabajo del nuevo estructuralismo (Lounsbury and Ventresca, 2003) enfatizan los aspectos multiniveles sociales, culturales y políticos del comportamiento organizacional y del fenómeno. De acuerdo a los contestantes políticos más poderosos en la arena social será posible tener sus esquemas reflejados dentro de la lógica institucional.

\section{Teoría Institucional}

El institucionalismo de la primera mitad del siglo XIX tenía una orientación descriptiva y usaba el razonamiento inductivo. El viejo institucionalismo de Commons (1950) considera que las instituciones existentes en un tiempo determinado representan soluciones imperfectas y pragmáticas a los conflictos pasados. Así, la historia institucional es un proceso de selección de un conjunto de prácticas institucionales sobre un conjunto de alternativas en un proceso de toma de decisiones pragmáticas que involucran el descubrimiento a través de la investigación y la negociación de lo que es la mejor práctica en las circunstancias actuales de intereses organizados en conflicto, para imponer su voluntad colectiva entre los grupos y sobre los individuos. 
Los institucionalistas describen las instituciones como acción de gobierno en los campos organizacionales. Las instituciones son consideradas como recursos de los agentes y actores racionales para obtener el logro de sus objetivos. Las instituciones son esquemas, normas y regulaciones humanamente divisados que permiten y constriñen la conducta de los actores sociales y hacen predecible y significativa la vida social (Scott, 2001; North, 1990, DiMaggio and Powell, 1991).

La sociología política y el institucionalismo de la ciencia política fundamentaron conceptualmente la noción del buen gobierno empujando la instauración de procesos de gobernabilidad democrática y el análisis de los procesos de informalización de la política. El buen gobierno, esencia de la gobernabilidad democrática, se centra en los procesos de formulación y ejecución de políticas públicas creadoras y reguladoras de instituciones y mecanismos que permitan a los actores colectivos, acordar, negociar y asumir funciones de vigilancia de la esfera pública.

Existe un consenso emergente que relaciona los valores, ideas comunes, principios y normas que son sustentados por las instituciones estatales y no estatales que están involucrados en al gobernabilidad corporativa. La institucionalización de los principios de la gobernabilidad corporativa a través de la emisión de códigos tiene un impacto en las redes institucionales integradas que intentan regularizar las expectativas.Los instrumentos del institucionalismo se aplican a la ciencia política en el análisis de los procesos de disolución como una inevitable acción de diferenciación social latinoamericana. La sociología política describe la complejidad y la fragilidad de la inserción de la sociedad civil en la esfera pública y endereza las críticas hacia el modelo de desarrollo neoliberal impuesto por el estado.

Existe un amplio consenso de que la teoría institucional ofrece un acercamiento poderoso para el estudio de la administración internacional, ha sido sorprendente que se gaste poco tiempo en las discusiones sobre qué tipo de acercamiento es el más apropiado. Un creciente número de académicos de la administración internacional están aplicando la teoría institucional al estudio de las multinacionales ya que provee un fundamento teórico rico para examinar un rango amplio de temas críticos y permite la teoría en múltiples niveles de análisis esencial para las investigaciones de las multinacionales. Como acercamientos críticos a la administración internacional ganan ascendencia en la literatura de los estudios organizacionales (Peltonen, 2006) los investigadores llegan a ser crecientemente conscientes que la teoría acerca de las diferencias del poder entre las unidades de las multinacionales ente el occidente y el no-occidente es esencial para determinar la naturaleza contestada de los procesos de la administración internacional.

Algunas de las aplicaciones fundamentales de la teoría institucional en la literatura de la administración internacional, comprenden la conceptualización de los ambientes nacionales en términos de los pilares regulatorios, normativos y cognitivos introduciendo constructos tales como perfiles institucionales del país, para conceptualizar los procesos de transformación en gran escala de los sistemas nacionales a través de las nociones de transición institucional, levantamiento e imperfección, para explicar los sistemas de negocios nacionales comparativos basados en el involucramiento institucional, para explicar las similitudes en las prácticas a través de las organizaciones que resultan de las presiones isomórficas, para explicar las restricciones en la difusión e institucionalización de prácticas en las organizaciones a través de las fronteras y unidades de las multinacionales y explicar las relaciones entre las multinacionales y sus ambientes anfitriones basados en las nociones de legitimidad y posibilidad de lo extranjero.

El institucionalismo organizacional examina las adaptaciones y conformaciones de las organizaciones a las presiones del medio ambiente institucional para conseguir la legitimidad (DiMaggio and Powell, 1983, 1991; Scott, 2001). Estos autores han analizado la difusión de instituciones entre organizaciones en poblaciones a través de procesos evolutivos de variación, selección y retención de instituciones típicamente prácticas y formas organizacionales.

El papel de los agentes sociales en el contexto de las multinacionales consistentes con el viejo institucionalismo, las preferencias del actor son influenciadas por los procesos de socialización. Implican normas y valores que surgen en los lugares nacionales o localizados. Una implicación de los mercados dentro 
del contexto institucional e ideológico es que no son construcciones de la teoría económica abstracta y histórica, sino que nunca pueden ser libres. El contexto institucional provee oportunidades para explorar la construcción de las teorías más que una orientación explotativa donde se apliquen constructos, teorías y métodos ya aceptados.

Hay varios cuerpo de la literatura relacionados con el institucionalismo que son instructivos para desarrollar más formas de tratar los temas teóricos tales como la emergencia de las instituciones, cómo las organizaciones dan sentido a sus ambientes institucionales complejos, cómo las organizaciones activamente se posicionan así mismos dentro y fuera de los campos meta, meso e intra, cómo la agencia tienen lugar, como se implica la agencia, cuales son las fuerzas motivadoras que orientan la similitud organizacional y cómo las organizaciones sobreviven dada la complejidad de las limitaciones sociales que enfrentan. No se puede contar con los externos para compromisos creíbles (Williamson, 1996:50) deben ser monitoreados, una conclusión que la economía d los costos de transacción comparte con la teoría de la agencia en donde los agentes son vistos como partes autointeresadas (Kim, Prescott and Kim, 2005).

La cultura no juega un papel relevante en estas teorías y solo es incorporada en algunos de los supuestos. Estas teorías han sido eclipasadas $\mathrm{n}$ los años recientes por las economías de los costos de transacción (Williamson, 1975, 1985) que se ha convertido en la base teórica predominante. La distancia cultural emergió como medida y metáfora al mismo tiempo que la teoría de los costos de transacción (Williamson, 1979). Las metáforas, las teorías y los métodos pueden tener una existencia simbiótica. La aceptación incondicional de la metáfora de la distancia cultural fue alentada por la prevalencia de las teorías de los costos de transacción y la agencia, estrechas y positivas. Críticos del reduccionismo cultural de la teoría de la distancia cultural avanzan la noción de distancia institucional (Kostova and Roth, 2002) como una forma alternativa de medir las similitudes y diferencias entre las instituciones regulatorias, cognitivas y normativas. Claramente la orientación cultural desde esta perspectiva de organización como el resultado de una falla del mercado exento de cualquier ramificación del poder (Hofstede, 1983, 1993, 1994, 1996), la teoría de los costos de transacción no trata directamente de la cultura o de la inversión extranjera.

Algunos paralelismos son evidentes entre el concepto de hegemonía y la isomórfica estabilidad de la teoría institucional. En ambos el orden social es visto como contingente en un balance de la presión coercida de reglas y más fuerzas consensúales de normas, marcos de referencia cognitivos e ideas que se dan por dadas. Los procesos de construcción o de retos a la hegemonía corresponden al modelo político de la acción en los campos organizacionales (Fligstein, 1997:398) quien apunta a la importancia de los actores para mantener una imagen de carencia de sentido de sí mismos para enmarcar los asuntos en las formas que resuenen con las concepciones de interés a fin de construir coaliciones más amplias. El modelo de acción colectiva enfatiza la importancia del conflicto, poder y política en sus implicaciones en la innovación institucional. Alvarez, Mazza, Pedersen and Svejenova (2005) han avanzado a una teoría compleja de la acción.

Estos conceptos incorporados a los estudios del cambio institucional, todavía prometen mayores desarrollos de investigación por los institucionalistas. Los institucionalistas analizan el conflicto, el poder y la política en el cambio institucional. También describen los esfuerzos intencionales de los actores institucionales que afectan el cambio institucional. El cambio institucional se define como una diferencia en forma, cualidad o estado en 1 tiempo en una institución. El cambio en un arreglo institucional puede ser determinando observando el arreglo en dos o más puntos en el tiempo en un conjunto de dimensiones institucionales.

Los procesos de cambio institucional son con frecuencia procesos políticos de movilización de campañas para legitimar la innovación social y etnológica en las organizaciones. Braithwait and Drahos /2000) proponen modelos de procesos de cambio institucional considerando que el cambio es una característica precipitada por la ocurrencia de la significación o disfunción de un evento que dispara la apreciación o amenaza la nueva oportunidad.

Clemens and Cook (1999) desarrollaron un tratamiento teórico del cambio institucional que integra modelos de cambio estructurales, ecológicos y de procesos dialécticos y argumentan presentando evidencias empíricas, que 
las instituciones no siempre se reproducen confiablemente, dependiendo de variables como las características de las redes sociales, el aprendizaje de los actores, las contradicciones y multiplicidad de las instituciones, etc. Los arreglos institucionales en los que el cambio institucional tiene lugar, así como en los esfuerzos de los activistas sociales y de los emprendedores tecnológicos alientan estos cambios.

El concepto de campo deriva de la atención de la teoría institucional al comportamiento de las organizaciones dentro de las redes interrelacionadas. Los campos organizacionales consisten de agencias reguladoras, sociedades profesionales, consumidores, proveedores, y organizaciones que producen bienes y servicios similares que exhiben distintas reglas de juego, redes relacionales y distribuciones de recursos (Rao, Morril, and Zald, 2000: 251). Las teorías institucionales explican la convergencia y la estabilidad en los campos en términos de fuerzas isomórficas regulativas, cognitivas y normativas. Los teóricos institucionales han enfatizado la naturaleza conflictiva de la contestación de los actores sobre las estructuras y procesos del campo (Macguire et al, 2004). El análisis de Hoffman (1999: 352) sobre las prácticas ambientales en competencia y los marcos de referencia discursivos enfatizan como los campos constituyentes son con frecuenta armados con perspectivas opuestas más que con retóricas comunes en un proceso que puede asemejar más la guerra institucional que un diálogo isomórfico.

Los retos al institucionalismo en el contexto de las multinacionales responden más al nivel meso de análisis y menos a los niveles meta e intra. Por tanto, la alternativa es mezclar los niveles de los procesos institucionales lo cual es más relevante que el nivel meso. Es más apropiado bajo condiciones de ambigüedad institucional y contradicciones que el nivel meso y aunque es válido lo es menos cuando se aplica a los niveles de análisis meta e intra, debido al ambiente de negocios internacional tradicionalmente débil. Ya que el nivel meso es exactamente donde la mayor parte de la investigación de las multinacionales se hace se debe considerar a las muiltinacionales en la investigación de la administración internacional e involucrarse menos en lo parroquial y más en la construcción de una teoría sofisticada intelectualmente dentro de la perspectiva institucional, lo cual requiere acercamientos multidisciplinarios y un cambio ontológico del positivismo puro y del empiricismo. La teoría institucional es vista como la fuente de los desarrollos teóricos mientras que administración internacional es relegada al papel de la aplicación de estas ideas.

Phillips and Tracey (2009) critican que los nuevos desarrollos en la teoría institucional han sido ignorados por los académicos de la administración internacional incluso cuando estos desarrollos presentan importantes ideas que pueden proveer mejores respuestas a las cuestiones institucionales en la investigación de las corporaciones multinacionales. Kostova, Roth and Dacin (2008) formalmente cuestionan las aplicaciones formales actuales en la investigación de las multinacionales a través de un conjunto de provocaciones que responden a los límites del contexto de las multinacionales en la teoría institucional, particularmente la nueva perspectiva que ha estado dominando la investigación de la administración internacional.

Se cuestiona si la teoría institucional es útil para la investigación en la administración internacional, y si estas ideas son válidas en el contexto de las multinacionales, cuales aplican y cuales necesitan ser modificadas y requieren más desarrollo. Reconociendo estas distinciones, Kostova and Zhaheer (1999) ofrecen una teoría especial sobre la legitimidad de las multinacionales, argumentando que es necesario porque las multinacionales enfatizan la condición de complejidad en la legitimación del ambiente externo, el ambiente intraorganizacional y los procesos de legitimación.

Los académicos pueden cuestionarse cual es la mejor conceptualización que reconozca las áreas de la vida institucional de las corporaciones multinacionales mediante un punto de vista de campo organizacional más desarrollado y se apunta a nociones concretas en las cuales la noción de campo se reconsidere para incorporar el nuevo papel de actores diversos y más distribuidos, los temas de la agencia y la contextualización, las herramientas cognitivas tales como los guiones, esquemas y tipificaciones. La teoría sugiere que el campo organizacional es un concepto útil para entender el ambiente institucional que enfrentan las corporaciones multinacionales en los contextos institucionales en los que operan (Phillips and Tracey, 2009:170). Tratar de 
estos temas es la forma primaria en que la administración internacional debe ser capaz de aplicar y moverse hacia la perspectiva de la teoría institucional.

Se critica a la teoría institucional que ha sido aplicada al estudio de las corporaciones multinacionales. Kostova, Roth and Dacin (2008) consideran que la investigación se ha quedado corta en determinar las implicaciones teóricas del contexto de las multinacionales y la utilización de la distinción de las organizaciones mantiene un potencial para fortalecer la construcción de la teoría en esta área, pero la naturaleza de las multinacionales eleva cuestionamientos fundamentales acerca de la validez de esta perspectiva para este contexto, específicamente lo significativo que son las nociones de campo organizacional, isomorfismo, legitimidad y descoplamiento cuando se consideran a las multinacionales.

Las corrientes del institucionalismo liberal y construtivismo retan a la interpretación realista. La ingeniería institucional que impulsa el liberalismo es muy sugerente. Los institucionalistas liberales difieren de los realistas en los prospectos para la creación y mantenimiento del régimen. Para los liberales, los regímenes son creados por los Estados con frecuencia comparten fuertemente temas mutuos en áreas temáticas específicas (Zacher and Sutton, 1998:3). Estos regímenes pueden modificar las preferencias del Estado mediante la creación de foros para la negociación internacional como mecanismos para la resolución de conflictos. Pero las instituciones liberales no son perfectas ni coherentes en sus reglas, normas, leyes, costumbres, tradiciones, usos morales, etc. En las instituciones liberales se mezclan la legalidad y la legitimidad, las leyes y moral, las normas y las costumbres, etc., las cuales en ocasiones derivan en disfuncionalidades económicas, políticas y sociales.

Las corrientes del institucionalismo liberal y construtivismo retan a la interpretación realista. Los institucionalistas liberales difieren de los realistas en los prospectos para la creación y mantenimiento del régimen. Para los liberales, los regímenes son creados por los Estados con frecuencia comparten fuertemente temas mutuos en áreas temáticas específicas (Zacher and Sutton, 1998:3).

Dadas las muchas limitaciones sin embargo, los académicos no deben abandonar la teoría institucional que mucho como perspectiva, aunque los académicos en la administración internacional deben alejarse de las pocas ideas básicas institucionales que han sido usadas continuamente e indiscriminadamente. Estas ideas han limitado la validez y requieren una seria reconsideración teórica de las multinacionales. Kostova, Roth and Dacin (2008) sugieren que las nociones básicas de campos, isomorfismos, desconexión y legitimidad necesitan ser modificadas dada la naturaleza de las multinacionales y proponen que los académicos en la administración internacional deben desarrollar aplicaciones de la teoría institucional más sofisticada para el estudio de las multinacionales mediante la incorporación de una más amplia literatura institucional.

\section{Neoinstitucionalismo}

El neoinstitucionalismo que aglutina enfoques históricos, sociológicos y de la elección racional, surge a principios de la década pasada como un conjunto de reglas que determinan los procesos de la reforma institucional a partir de los marcos de incentivos y restricciones impuestos a los comportamientos de los diferentes agentes y actores económicos, sociales y políticos para la formulación e implantación de políticas públicas y que tienen un impacto en los resultados medidos en términos de crecimiento y desarrollo. Las primeras interpretaciones de la teoría del nuevo institucionalismo se enfocaron directamente en el isomorfismo y la legitimación pero un cuerpo significativo de los más recientes trabajos ha demostrado una fuerte y sostenido interés en agencia y cambio. Oliver (1991) reorienta la falta de atención a la agencia humana del neoinstitucionalismo temprano combinó la teoría institucional con la teoría de la dependencia de los recursos para desarrollar una tipología de respuestas estratégicas a las presiones del ambiente.

El neoinstitucionalismo estudia los rasgos de las estructuras institucionales económicas que posibilitan el desarrollo de los pueblos. De acuerdo a Burgos Silva (2002), el análisis económico neoinstitucionalista define la institucionalidad jurídica y el desarrollo económico, cuestiona el derecho como instrumento del desarrollo 
económico y reconoce a las instituciones informales y promueve mecanismos institucionales considerados como óptimos. La teoría neoinstitucionalista argumenta que la importancia de los marcos de referencia normativos y las reglas de comportamiento para guiar, constreñir, y crear poder en las organizaciones las que se consideran consisten de estructuras y actividades cognitivas, normativas y regulativas que dan significado al comportamiento social. Los análisis en ciencia política desde la perspectiva del nuevo institucionalismo a partir de las consideraciones de la elección racional de los individuos a los incentivos que ofrecen las instituciones entendidas como las reglas que prescriben, proscriben y permiten los comportamientos.

El neoinstitucionalismo enfatiza las instituciones que definen el comportamiento de los actores frente a su medio social. El neoinstitucionalismo económico analiza las fallas de los mecanismos del Estado y sus ineficacias. La economía neoinstitucional demuestra las deficiencias e incapacidades del Estado como mecanismo de gobernabilidad y coordinación que garanticen los acuerdos y compromisos sobre la propiedad. El neoinstitucionalismo económico relaciona en forma inextricable la economía y la política y analiza las fallas de los mecanismos del Estado y sus ineficacias. De acuerdo a la teoría neoinstitucional de la economía, el subdesarrollo es resultado de las fallas del Estado para proveer las estructuras de governance necesarias para garantizar las instituciones que apuntalan el desarrollo de los pueblos. El neoinstitucionalismo recomienda un Estado fuerte pero limitado en sus funciones. El contenido del Estado es sólo garantizar al mercado la posibilidad de ejercer su función sin obstaculizar su trabajo y protegerlo de injerencias ajenas (Estefanía, 2002).

El papel de los valores es central al viejo institucionalismo pero el neoinstitucionalismo se orienta más a los procesos cognitivos. Greenwood and Hinings (1996:1022) resumen este cambio señalando que el viejo institucionalismo enfatiza los temas de influencia, coaliciones y los valores de competencia fueron centrales, junto con el poder y las estructuras informales y el nuevo institucionalismo que enfatiza la legitimidad, el involucramiento de los campos organizacionales y la centralidad de la clasificación, rutinas, guiones y esquemas. El nuevo institucionalismo atiende a los campos organizacionales como unidades de análisis. Los procesos institucionales pueden dar cierta estabilidad a los campos organizacionales, aunque estos siempre están evolucionando y no son estáticos, resolviendo mediante consenso negociado socialmente las diferencias de interpretación.

El modelo de diseño institucional sustentado en la teoría neoinstitucional (Barley and Tolbert, 1997) y la agencia humana adaptado del modelo de la estructuración, argumentan que los actores pueden escoger conscientemente revisar más que replicar las instituciones existentes. El nuevo institucionalismo se cimienta en un individualismo metodológico que se fundamenta en el principio de que todos los resultados de las acciones humanas se explican por la acción individual cuyas interacciones en las estructuras legitiman las instituciones. Este individualismo metodológico tiende a incentivar a los individuos en función de sus acciones. El neoinstitucionalismo pretende ordenar la esfera pública bajo un esquema de instituciones orientadas más al ordenamiento privado que aniquila toda pretensión de igualdad económica, social y política, a partir del supuesto de que ya han sido obtenidas por el régimen democrático.

Entre el enfoque neoinstitucionalista y el de regulación democrática emerge el concepto de gobernabilidad como una relación existente entre los procesos de libre mercado y los procesos de la democracia. El neoinstitucionalismo se fundamenta más en el razonamiento deductivo y se expresa en dos grandes enfoques: en la elección de las estructuras de governance de los actores privados en un determinado medio ambiente que se convierte en el objeto de análisis económico y en el cambio institucional en función de los efectos que los diferentes medios ambientes institucionales tienen en el desempeño económico y en el desarrollo de las instituciones con el apoyo de modelos mentales compartidos e ideologías.

La mayor parte de los académicos de la administración internacional tienen un punto de vista estrecho sobre la teoría institucional centrada más en el neoinstitucionalismo (Meyer and Rowan, 1977) y utilizando los conceptos de campo organizacional, legitimidad, isomorfismo y mecanismos de presiones internacionales. El modelo neoinstitucional esencialmente mantiene que la sobrevivencia organizacional se determina por el grado 
de alineación con el ambiente organizacional y por tanto, las organizaciones tienen que cumplir con las presiones organizacionales externas.

Una revisión a las aplicaciones de la teoría institucional en la administración internacional identifica los temas que han sido estudiados y las principales ideas institucionales usadas, las cuales ha sido dominado por un estrecho subconjunto de ideas institucionales que primeramente vienen del neinstitucionalismo. Si se permite una cantidad nominal de agencia, el neoinstitucionalismo sugiere que la incorporación de elementos institucionales permite a los actores organizacionales llevar la organización como legítima y por tanto, alentando su probabilidad de sobrevivencia.

Conclusiones similares se derivan del trabajo de Lawrence and Phillips (2004) quienes sugieren que el cambio institucional en la forma de arreglos del nuevo institucionalismo resultan no solamente del contexto macro histórico exógeno sino también de la acción de los emprendedores institucionales. El concepto de emprendedor institucional es central a la teoría del nuevo institucionalismo pero permanece institucional al análisis institucional en la administración internacional. Este concepto tiene relevancia para las multinacionales que buscan operar en contextos diferentes y deben llegar a ser un tópico principal en la administración internacional. La internacionalización trae el poder de las firmas como agentes del cambio institucional.

Kostova and Dacin (2009) argumentan que los académicos de la administración internacional vienen usando un subconjunto muy estrecho de ideas neoinstitucionales que no tienen el potencial para manejar la complejidad del contexto de las multinacionales y no reflejan los nuevos desarrollos en la teoría institucional. Kostova, Roth and Dacing (2008:997) claman que los académicos de la administración internacional se fundamentan en un punto de vista de la dinámica institucional de la teoría del nuevo institucionalismo que esencialmente sostiene que la supervivencia de la organización está determinada por el grado de alineamiento con el ambiente institucional mientras que permite una cantidad nominal de la agencia, los institucionalistas sugieren que la incorporación de los elementos mandados permite a los actores organizacionales portar a la organización como legítima y por tanto alentando las posibilidades de sobrevivencia. Esta crítica se refiere a una versión de la teoría institucional.

Kostova, Roth and Dacing (2008:1003) concluyen que la teoría del nuevo institucionalismo tal como es actualmente constituida no es apropiada para el estudio de las multinacionales y proveen críticas específicas. (Greenwood and Hinings, 1996:1024) proponen que le viejo y el nuevo pueden ser combinados a fin de proveer un modelo de cambio que conecta el contexto organizacional con la dinámica intraorganizacional. Kostova, Roth and Dacin (2008) desarrollan un conjunto de provocaciones que retan la validez del neoinstitucionalismo tradicional y ofrecen ideas en una teoría más novedosa basada en la integración del viejo y el nuevo institucionalismo. Todas las unidades en las multinacionales pueden ser vistas como perteneciendo al mismo campo institucional intraorganizacional el cual se contiene dentro de las fronteras de la firma. Este campo puede ser más fuerte en ejercitar influencia sobre los miembros, esto es sobre las subunidades multinacionales que el campo externo tradicional discutido en el neoinstitucionalismo.

Kostova, Roth and Dacin (2008) contienden que las multinacionales ser involucran en menos desconexiones y ceremoniales que los sugeridos típicamente por los neoinstitucionalistas. Kostova, Roth and Dacin (2008) consideran que teóricamente estas condiciones son mejor entendidas si las ideas del viejo institucionalismo se mezclan con los puntos de vista neoinstitucionales. Opuesto al énfasis neoinstitucional sobre estática, resultados, cognición y el dominio y continuidad del ambiente, el viejo institucionalismo se enfoca en la dinámica, la construcción del cambio social, y los valores y enfatiza un punto de vista dominado por la agencia (Hirsch and Lounsbury, 1997:406).

Phillips and Tracey (2009) presentan los recientes desarrollos de la teoría del nuevo institucionalismo y sugieren que no es que la teoría del nuevo institucionalismo no sea relevante a la administración internacional como lo es la forma de la teoría institucional adoptada en las primeras ideas del nuevo institucionalismo y especialmente el de Scott (1995). No debe abandonarse la perspectiva neoinstitucional sino más bien concurrir con muchos sociólogos y se sugiere que el viejo y el nuevo institucionalismo deben traerse juntos para el 
estudio de las multinacionales introduciendo elementos para un acercamiento que pueden ser considerados como una réplica inicial a las provocaciones de Kostova, Roth and Dacin (2008) y como los fundamentos de una teoría más refinada acerca de las multinacionales.

Phillips and Tracey (2009) construyen sobre los desarrollos recientes de la teoría institucional para cuestionar los argumentos de Kostova, Roth and Dacing (2008) y critican su discusión sobre el concepto del campo organizacional y argumentan por la utilidad del concepto de entrepreneur institucional y están de acuerdo en que la conceptualización de la teoría institucional en la administración internacional es inadecuada pero sugieren algunas formas alternativas de pensamiento.

Los académicos de la administración internacional ignoran el llamado a integrar el viejo y el nuevo institucionalismo que puede representar una promesa para el estudio de las corporaciones multinacionales (Greenwood and Hinings, 1996). Phillips and Tracey (2009) sugieren que los investigadores de la administración internacional se benefician de la emergencia de la mezcla de las dos corrientes del viejo y el nuevo institucionalismo enfocado en cómo los actores ejercitan la agencia para crear estructuras de nuevo institucionalismo y alterar las existentes que están difusas a través de los campos organizacionales. Sumarizan esta posición señalando que una consideración más sostenida del trabajo reciente de la teoría institucional que trate con la agencia y el cambio institucional así como con el isomorfismo y la legitimidad.

Phillips and Tracey (2009) consideran como puntos importantes que la administración internacional debe beneficiarse del trabajo reciente de la teoría institucional, el concepto de campo organizacional provee un marco de referencia útil y el estudio de las multinacionales beneficia en forma más desarrollada y consistente el uso del concepto, y el concepto de emprendimiento institucional es central en la teoría del nuevo institucionalismo pero permanece marginal en los análisis institucionales de la administración internacional. Estos tres puntos implican un punto de vista consistente acerca de la relación intelectual entre los campos de la teoría institucional y la administración internacional. Phillips and Tracey (2009:169) sugieren que los investigadores de la administración internacional se benefician de los recientes avances en la teoría institucional.

Phillips and Tracey (2009) sugieren que las necesidades de los investigadores de administración internacional es tomar un punto de vista amplio sobre la teoría institucionalista y sus recientes desarrollos a fin de reflejar el amplio cuerpo de investigación que ha surgido desde los trabajos de Scott (1995) y que los primeros institucionalistas, tales como Meyer and Rowan (1977) and DiMaggio and Poweel (1983) integra una preocupación igual por la agencia y el cambio con la legitimidad, la estabilidad y el isomorfismo. Estos trabajos recientes integran una preocupación igual de la agencia y el cambio y enfocan su atención en las dimensiones simbólicas de las instituciones y enfatizan un simbolismo de raíces de constructivismo social de la teoría institucional, los cuales son desarrollos teóricos que tienen un gran potencial para los investigadores de la administración internacional y para entender las dimensiones institucionales de las corporaciones multinacionales.

Kostova and Dacin (2009) proponen que las ideas recientes del institucionalismo, tales como la agencia activa, el emprendurismo institucional, la estrategia del discurso y las contradicciones institucionales y las prácticas mantienen la promesa para la administración internacional, pero la aplicación del trabajo institucional reciente es solamente parte. Estos esfuerzos del emprendurismo institucional a los campos de cambio se encuentran frecuentemente con resistencias de los campos dominantes definidos por McAdam and Scott (2005: 17) como aquellos individuos, grupos y organizaciones cuyas acciones e intereses el campo tiende a resolver. Particularmente instructivo es el trabajo del emprendurismo institucional (Lawrence and Phillips, 2004), estrategias retórica y discursivas (Suddaby and Greenwood, 2005) y contradicciones y prácticas institucionales así como la teoría sobre la creación de ambientes institucionales.

Debido a la distinción conceptual, el contexto de las multinacionales debe ser usado para desarrollar ideas institucionales nuevas. Kostova and Dacin (2009) consideran que la academia de la administración internacional debe usar el único contexto de las multinacionales para cuestionar los supuestos de las 
condiciones de frontera de la teoría institucional tradicional creando las posibilidades de nuevos desarrollos significativos en el pensamiento institucional de tal forma que no solamente retornen algo mejor a la literatura sino que también generen algo nuevo que enriquezca la perspectiva teórica empleada.

Una crítica al neoinstitucionalismo es que diseña arreglos institucionales con criterios óptimos de implantación en los países más desarrollados, los cuales son seriamente cuestionados por el path dependence para ser adaptados en los países menos desarrollados. Es cuestionable en la teoría económica neoinstitucional el hecho de que las instituciones disfuncionales que no alcanzan los niveles óptimos, permanezcan durante mucho tiempo y prolonguen el subdesarrollo, debido a causas fundamentalmente de mecanismos de auto-refuerzo en procesos de dependencia de recursos. La teoría de la dependencia de recursos relaciona la acción al poder señalando que la organización que controla recursos de los cuales otras organizaciones son dependientes, son capaces de influenciar estos otros.

\section{Constructivismo social y la perspectiva crítica interpretativa}

Las reacciones a las tendencias institucionales y en especial al modelo de elección racional en los ochentas y noventas fueron hacia los modos del constructivismo y el postmodernismo dentro del campo de la administración internacional. El constructivismo ha emergido en años recientes como una respuesta parcial a las limitaciones de la concepción racionalista dominante. El criticismo de que se entiende poco de donde vienen las normas ha sido denominado generalmente constructivismo (Checkel, 1998).

Para los constructivistas, las normas se refieren a los elementos intersubjetivos y las reglas son usadas por los racionalistas para referirse a los elementos formales. La otra característica fundamental de los elementos intersubjetivos de las instituciones internacionales que repiten sus cualidades deónticas evaluativos o normativas. Las normas son creencias compartidas acerca de cómo las cosas deben ser hechas o cómo las cosas deberían hacerse. La literatura constructivista se enfoca en la persuasión y la socialización como los factores clave del proceso de reconstrucción de normas.

Los constructivistas tratan de explicar cómo y por qué los significados se institucionalizan o cambian, analizan cómo las instituciones compuestas de reglas y normas, prácticas y procedimientos habitualmente establecidas. Los constructivistas pueden orientarse hacia una base ética más amplia de los individuos y ser capaces de rendir cuentas para replicar o confrontar las reglas del juego. Los constructivistas confrontan el supuesto de que los Estados han sido los actores primarios en un sistema internacional anárquico y abre las posibilidades para la agencia y el cambio. Los constructivistas están de acuerdo en interpretar la naturaleza de las instituciones sociales, las cuales pueden ser categorizadas en modernas o postmodernas, positivistas o post-positivistas.

Para los constructivistas, que estudian las normas y los discursos como componentes de las más amplias instituciones sociales y algunos se enfocan en las posibilidades de cambio, la estructura son los patrones de orden social institucionalizados pero no inmutables que reflejan el contexto histórico. Las instituciones sociales son vistas como consistentes de normas o conjunto de normas, las cuales son definidas por los constructivistas como expectativas socialmente compartidas, entendimientos o estándares de conductas apropiadas por actores con una identidad dada (Boekle. Rittberger and Wagner, 2001:106).

Las identidades y las normas enfatizadas por el constructivismo pueden proveer estabilidad cuando son constantes, pero pueden llegar a ser una fuente del desorden cuando son están fluyendo. Así por ejemplo, las identidades son condicionadas por el contexto cultural, más que determinadas por cualquier característica objetiva. De los significados y prácticas se pueden esperar determinaciones e indeterminaciones. En los entendimientos intersubjetivos de representaciones, normas, discursos, reglas, etc., los constructivistas demuestran que ideas dominan.

El constructivismo explora el grado en que los actores refuerzan o trascienden las prácticas institucionalizadas y procesos de legitimación, aprendizaje, razonamiento y otras formas de comunicación, reforzando o recreando 
los significados institucionalizados en las estructuras sociales. Este proceso de legitimación es diferente al estrecho descrito en las teorías neoinstitucionalistas. Los actores (Estados, individuos, organizaciones, etc.) actúan para perseguir estrechamente sus propios intereses, aunque los tres enfoques difieren en sus consecuencias.

Los constructivistas se enfocan en el rol de ideas, normas, conocimiento, cultura, y argumento en política enfatizando en particular el papel colectivamente sostenido de ideas ínter subjetivas y entendimientos en la vida social (Finnemore and Sikkink, 2001:392). Los constructivistas describen las normas como expectativas compartidas o estándares de conducta apropiada. Además, las instituciones pueden constituir actividades de categorías de acción, aunque muchas formas de conducta de naturaleza física tienen un papel en determinar su significado social. Analíticamente distinto de la creación de actores es el papel que las instituciones pueden jugar en determinar sus identidades, intereses, metas y preferencias el cual es un tema prominente en la literatura constructivista sobre las normas.

El constructo teórico a nivel macro, el acercamiento constructivista social particularmente los desarrollos relacionados con el ciclo de vida de la norma (Finnemore and Sikkink, 1998). Miller and Edwards (2001) enfatizan la formas en la cuales las normas pueden ser introducidas por los emprendedores para atraer una masa crítica, y como algunas normas son eventualmente internalizadas. La concepción constructivista pone énfasis explícito y primario en el aspecto intersubjectivo de las instituciones internacionales y relacionan las instituciones como involucrando ideas que son compartidas por todos los miembros de una colectividad y fenómenos fundamentalmente ideacionales, involucrando ideas que son compartidas por todos los miembros de una colectividad. Pero los conceptos ideacionales tales como el discurso, creencias, normas, no capturan las relaciones de intersubjetividad entre los agentes y las estructuras que distingue el constructivismo.

Se definen estos entendimientos intersubjetivos en formas diferentes. Para algunos, las normas proveen estándares explícitos de conducta mientras que otros enfatizan las reglas que toman formas linguísticas y no lingüísticas. Para otros, el discurso relaciona significados a acciones. Pocas instituciones pueden ser completamente ideacionales en la naturaleza, pero los constructivistas han hecho un caso para verlas como intersubjetivas porque hasta cierto grado, las instituciones existen en las mentes de las gentes y no necesariamente necesitan estar escritas en alguna parte sino que se caracterizan por ser modelos mentales compartidos.

Los constructivistas típicamente distinguen entre dos principales tipos de normas, las regulatorias o prescriptivas, las cuales son similares a las normas expresadas en la concepción racionalista en tanto que ordenan y constriñen la conducta. Las normas y reglas regulativas u operacionales pueden ser prescriptitas, que requieren u obligan a los actores a actuar en ciertas formas, Es precisamente la cualidad evaluativo o prescriptiva del deber ser la que establece normas aparte de otros tipos de reglas.

La teoría constructivista asume que los Estados no son solo actores sociales sino que son actores sociales capaces de monitorear reflexivamente sus acciones por lo que buscan legitimar sus estructuras sociales, una búsqueda que es difícilmente un proceso lineal o evolutivo. Los constructivistas promueven un punto de vista de agentes como actores con un propósito que buscan estructuras para dar significados a sus acciones. El enfoque de los constructivistas en la reflexividad y contingencia significa que la reflexividad sea vista como un movimiento positivo que mejora la condición humana, no tiene que ser tratada de esta forma. En la noción de la agencia los seres humanos importan porque son ellos quienes modelan o tienen la capacidad para cambiar la realidad social. Los seres humanos o agentes no existen aislados de las estructuras que crean, sin embargo, el constructivismo comparte el supuesto de que los agentes y estructuras son mutualmente constitutivas (Coconstitución). No se debe privilegiar uno a expensas del otro. A pesar de que los diferentes constructivistas tienden a enfatizar uno u otro.

La contingencia trabaja en cualquier forma y la innovaciones sociales pueden buenas y malas. En segundo lugar, la epistemología, a diferencia del constructivismo y la investigación de la paz democrática liberal falta en reconocer la acumulación del sujeto y el objeto o los agentes del Estado usan la teoría para informar de sus 
acciones. Para el constructivismo es posible la transformación del sistema por lo que las instituciones, intereses e identidades no son inmutables. Recientemente los académicos han conectado el constructivismo al idealismo liberal y han denominado a los constructivistas como teóricos liberales debido a que los realistas aplican una etiqueta de idealista al constructivismo, los empíricos de la elección racional son motivados por los supuestos constructivistas y ciertos constructivistas han intentado construir puentes en términos epistemológicos con los racionalistas (Steele, 2007).

El acercamiento construccionista social coloca a las metáforas dentro de un amplio contexto de relevancia social y encuentro jerárquico. Adoptando un acercamiento constructionista social se propone la fricción cultural como una metáfora substituta centrada en el encuentro actual de sistemas culturales dentro de un contexto de relaciones de poder y conflicto potencial entre una empresa multinacional y las comunidades del país anfitrión. Las multinacionales se involucran en manipulaciones específicas de los actores y en la negociación de su estatus dirigido a la construcción social de su aceptación y aprobación. La legitimidad es más una construcción social que una función de isomorfismo. La construcción de la imagen simbólica se convierte en crítica. Más recientemente la teoría y la investigación sobre la administración simbólica muestra que los líderes algunas veces influencian la toma de decisiones y la conducta a los grupos constituyentes externos involucrando en acciones o comunicaciones públicas que tienen la apariencia de conformidad a la lógica institucional o a las normas prevalecientes de la conducta corporativa.

Sin embargo, las teorías organizacionales tienden a enfatizar la construcción social de campos y por tanto, la importancia de la contestación política en los ámbitos cognitivos y normativos. Hardy and Phillips (1998:218) contienden que los campos emergen cuando diferentes organizaciones se perciben así mismas para estar conectadas en asuntos que le son comunes. No son estructuras objetivas predeterminadas sino procesos de construcción social y creación de significados en donde se negocia el orden social. Los teóricos críticos de la administración sugieren que los investigadores de la administración internacional adoptan en sus estudios el método crítico interpretativo que mantiene una ontología constructivista y una epistemología constructivista (Jack and Westwood, 2006: 486) que deben describir, analizar y abrir el escrutinio a las agendas ocultas, a los centros del poder y a los supuestos que inhiben, reprimen o constriñen.

Los proponentes del construccionismo social toman en cuenta la habilidad de una firma multinacional para emplazar actores astutos culturalmente para expandir conjunciones alrededor de los puntos clave de interdependencia con medio ambiente local pero al mismo tiempo examinan las actitudes del país anfitrión y de los actores del Estado hacia la cultura entrante y las herramienta que tienen para contar el impacto.

A pesar de la robustez de la perspectiva, la mayor parte de los estudios constructivistas de la administración internacional de la tecnología sobrepasan las realidades clave acerca de la transferencia de las tecnologías en las multinacionales. De acuerdo con la investigación del constructivismo social se demuestra que los usos de las nuevas tecnologías son el resultado de una compleja serie de interacciones entre los elementos materiales y los artefactos y las prácticas que constituyen los contextos nacionales y organizacionales en los que se implementan.

El constructivismo social da cuenta del cambio tecnológicamente inducido que niega la validez empírica de las determinantes tecnológicas. La relación entre cambio tecnológico y social es indeterminado fundamentalmente. Como una construcción social, el ethos del determinismo tecnológico llega a ser un poderoso mecanismo para crear un discurso que hace la relación indeterminada fundamentalmente entre la tecnología y la cultura para que aparezcan como que es determinada. Salen a relucir dos cuestiones importantes, cómo el discurso hace que surjan las relaciones indeterminadas para que aparezcan determinadas con la implementación de la nueva tecnología y cuáles son los beneficios y quienes se excluyen de los procesos de construcción social.

Adicionalmente, un enfoque en el uso del determinismo metodológico para crear un discurso poderoso que guie la acción organizacional puede extender el entendimiento teórico de la relación entre el mundo material y el discurso mostrando cómo materialmente puede ser enrolado discursivamente, traído hacia la acción humana para influenciar los procesos de construcción social. Los diseñadores y promotores de una tecnología no 
pueden predecir o controlar complemente sus usos finales. Siempre hay consecuencias no intencionadas y posibilidades anticipadas (Wajaman, 1995:199).

Las investigaciones que exploran las formas en las cuales el lenguaje influencia las prácticas con frecuencia tratan a las organizaciones como construcciones discursivas que representan al lenguaje que las personas usan cotidianamente. El uso de los discursos como un mecanismo de control a nivel de percepción es poderoso porque influencia los procesos de construcción social. Westphal and Clement (2008:889) examinan las determinantes y consecuencias de la influencia social y la reciprocidad en la relación entre los altos ejecutivos de una firma y un constituyente externo clave de las firmas, los analistas de seguridad que tienen una influencia significativa en la conducta de los analistas y otros miembros de la comunidad financiera hacia las firmas focales, su teoría y hallazgos de soporte trata finalmente de cómo los líderes corporativos pueden construir y perpetuar el apoyo externo para sus firmas mediante el involucramiento de procesos de influencia social que fomentan y mantienen intercambios sociales con los grupos constituyentes externos claves para las firmas.

El discurso administrativo sobre las implementaciones de las tecnologías administrativas internacionales muchas de las veces está fuera de los resultados empíricos generados por las calificaciones de los investigadores constructivistas. La administración internacional de la tecnología es vista como una lucha para establecer los discursos que ordenan y naturalizan al mundo de una forma particular. Entender por qué la implementación de la tecnología internacional algunas veces cambia la organización del trabajo en las multinacionales en formas predecibles aunque algunas veces no, requiere considerar como la tecnología induce los resultados que son creados discursivamente en los procesos de construcción social.

El análisis crítico problematiza los recuentos constructivistas sociales existentes de los procesos de cambio demostrando que los resultados inducidos tecnológicamente en las multinacionales no son simplemente construidos a través de las relaciones directas entre un individuo y una tecnología sino que los procesos de construcción son influidos hasta cierto grado por los discursos que dan forma a las percepciones individuales acerca de la tecnología en primer lugar.

Los acercamientos al constructivismo social sugieren que las resistencias al discurso de la inevitabilidad es posible porque la gente puede encontrase así misma en las cuales usan una tecnología en formas diferentes a lo que pretenden los diseñadores y los administradores. Un discurso de inevitabilidad trabaja socialmente en la construcción de resultados con tecnología en formas predefinidas porque cierra los antecedentes culturales a los procesos de construcción social haciendo la construcción indeterminada entre la tecnología y la cultura que parezca de alguna forma antiética a la naturaleza, a la ciencia y al progreso.

A pesar de que la perspectiva constructivista social reconoce los resultados inducidos tecnológicamente como fundamentalmente indeterminados y con probabilidad de que emerjan en formas diferentes a través de las diversas unidades de las multinacionales, el acercamiento ha sido lento para considerar el papel que el discurso y el poder de la política refleja pueden influenciar los procesos de construcción social. Como consecuencia, aquellos que no son capaces de definir los procesos de construcción social del cambio de la tecnología inducida en los términos de los otros quienes son representados meramente como recursos consumibles para ser asimilados sin resistencias.

La perspectiva crítica interpretativa provee un marco de referencia para tratar estos asuntos en el acercamiento de constructivismo social. Con raíces en la tradición fenómenológica de la escuela de Frankfurt en los trabajos de Husserl (1960), Gadamer (1975), Habermas (1984). Una perspectiva crítica interpretativa que comparte los mismos fundamentos ontológicos con la tradición constructivista social. Deetz (1982) sugiere que la perspectiva crítica interpretativa asume que el mundo es fundamentalmente indeterminado y está diseñado para investigar experiencias predictivas más que experiencias que ya han sido objetivadas.

Los teóricos de la crítica interpretativa argumentan que el discurso es siempre un resultado de algunos niveles de lucha entre los partidos con intereses variados (Alvesson and Deetz, 1999). Una conceptualización de la perspectiva crítica interpretativa fundamentalmente de naturaleza indeterminada de la realidad se alinea con lo 
ontológico del acercamiento constructivista social. Atendiendo a la forma en las cuales la realidad social se estructura en relaciones determinadas lleva a la investigación crítica interpretativa a los análisis de temas de poder y política. El objetivo para articular preocupaciones explícitas con temas del poder y la ideología en la teoría académica de la administración y organización internacional. El mundo social se construye a través de la ideología creada por las articulaciones discursivas de los procesos de cambio y por tanto es una creación inherentemente política.

Estas dos perspectivas difieren productivamente aunque los proponentes del acercamiento constructivista social muestran que la relación entre una tecnología y una multinacional organizándose es fundamentalmente indeterminada. A través de la crítica interpretativa para aumentar los estudios de la administración de la tecnología internacional provee las herramientas para entender cómo las articulaciones discursivas del mundo a la nueva tecnología implementada. Leonardi (2008) explora el discurso tecnológicamente determinista que acompaña la implementación de las nuevas tecnologías en las unidades locales de las multinacionales que influencian los procesos de construcción social. En un intento para suplementar el acercamiento constructivista social, con estos elementos Leonardi (2008) emplea acercamientos generados por una perspectiva crítica interpretativa (Deetz, 1992) que sugiere que el estado del mundo indeterminado fundamentalmente es hecho para aparecer determinado como poderosos actores construyen un discurso que ordena y naturaliza el mundo en una forma que ya seas explícita o inadvertidamente promueve sus propios intereses.

Tomando el punto de vista de que la tecnología y la cultura se entrelazan a través de procesos de construcción social que son práctica y discursivamente situados Leonardi (2008) contribuye a que estas perspectivas críticas emergentes sugiriendo que la administración internacional de la tecnología puede ser vista como una lucha entre los usuarios contextualizados, las interacciones físicas con los artefactos particulares y administradores descontextualizados, las articulaciones discursivas de los resultados de las tecnologías a través de varias unidades de las multinacionales. Leonardi (2008) combina la perspectiva crítica interpretativa con el acercamiento del constructivismo social a la administración internacional de la tecnología para argumentar que a pesar de las relaciones entre las tecnologías y las corporaciones multinacionales es fundamentalmente indeterminado, el discurso ha avanzado acerca de cómo una nueva tecnología debe cambiar la organización social del trabajo a través de las fronteras nacionales que pueden muy bien producir relaciones determinadas que parecen estar causadas por la tecnología en sí mismas.

Más que disminuir estos discursos como empíricamente inapropiados, incorrectos y mal informados Leonardi (2008) considera las articulaciones de los impactos discursivos que el determinismo tecnológico puede tener en las prácticas de la determinación de la tecnología administrativa en las multinacionales bajo el acercamiento de la perspectiva crítica interpretativa desarrollada por Deetz (1992) una perspectiva que sugiere que las comunicaciones no meramente reflejan el mundo social sino que lo producen. En esta perspectiva, los discursos juegan un rol clave en constituir las relaciones entre los sujetos y los objetos y son con frecuencia usados instrumentalmente por actores poderosos tales como los administradores organizacionales para hacer que las relaciones de un mundo indeterminado aparezcan como fijas e inmutables. La consecuencia de este discurso es que naturaliza la experiencia posicionando a la persona en el mundo de una forma particular previa a que el individuo tenga cualquier sentido de elección.

Este movimiento privilegia las prácticas de aquellos capaces de producir los discursos típicos de los administradores occidentales y marginar a aquellos actores en las unidades locales de las multinacionales típicamente con poco poder y los empleados no occidentales por la estructuración discursiva de las experiencias con la nueva tecnología de tal forma que reduce la posibilidad de apropiaciones situadas de la tecnología que jalan en línea con los valores culturales de la comunicación y la interacción, Al hacerlo se aumenta la crítica emergente y discursiva (Vaara, Tienari and Laurila, 2006). 


\section{Investigación cualitativa}

El interés reciente de la administración internacional en la investigación cualitativa es una panacea de los problemas de la investigación cuantitativa que reproduce una separación de la teoría y el método que mitiga contra la reflexión epistemológica (Jack and Westwood, 2006) y alienta un tipo de positivismo cuantitativo. Una razón por la cual la investigación cualitativa es importante para los académicos de la administración es que puede proveer detalladas descripciones de las acciones actuales en los contextos de la vida real (Gephart, 2004:455).

La investigación cualitativa interpretativa que se enfoca en la producción de significados y contextos empleados por los actores sociales en los lugares (Gephart, 2004: 457) pueden ser instrumentados en la generación de la teoría del contexto que provee importantes acercamientos en la forma en que los significados y sus implicaciones son formados por las fuerzas contingentes sobre las contextuales. El primer obstáculo metodológico tiene que ver con la ausencia relativa de la investigación cualitativa dirigida explícitamente a generar teorías de contexto como opuestos a los hallazgos de investigación cuantitativa en la literatura de la administración. Para los investigadores cualitativos este cambio puede involucrar dar una mayor consideración a la investigación cualitativa orientada al contexto y cuando es relevante, integrar la orientación del contexto inherente en muchas de esta investigación en su propia teorización.

Al hacerlo, los investigadores cualitativos pueden poner especial atención en especificar y probar precisamente como los constructos situacionales o temporales son probables que vengan a entrar en el juego y cualquier mecanismo de mediación a través del cual estos constructos temporales o situacionales pueden ejercer sus influencias. Para los investigadores cualitativos este cambio implica un enfoque en el desarrollo de procesos de las teorías explicando como se desenvuelven los fenómenos y como estos procesos pueden por sí mismos ser la situación o contingentes en el tiempo.

Alternativamente implica un mayor enfoque en la investigación interpretativa dirigida a examinar como los significados que se otorgan a diferentes fenómenos organizacionales varían a través de las situaciones, marcos del tiempo y unidades sociales. Cuando la investigación cualitativa relevante es deficiente los investigadores cualitativos pueden considerar adoptar ejemplos teóricos aterrizados, observaciones de campo, entrevistas abiertas y métodos cualitativos de estudios basados en casos en sus primeros niveles, desarrollo de prehipótesis de su investigación como medios por los cuales entender mejor las contingencias situacionales o temporales que potencialmente dan forma a los fenómenos de interés. Otorgado es probable que implique algún grado de riesgo de parte de los investigadores cualitativos, ya que los revisores y editores de revistas científicas con frecuencia confunden el rigor con teorías de varianza más positivista y son con frecuencia rápidos para rechazar acercamientos que se desvían del camino más familiar.

El acercamiento ganado de esta pre-investigación puede resultar en incluir nuevos constructos relacionados con el contexto y dejar otros mencionan la re especificación del modelo para incluir posibles efectos entre los niveles en las formas descritas. Este acercamiento puede ofrecer una posible solución a la dicotomía emergente entre los investigadores cualitativos y cuantitativos en donde los investigadores cualitativos profundizan en un contexto en particular en donde no pueden ver los patrones alrededor mientras que los investigadores cuantitativos se enfocan en encontrar fenómenos genéricos que pueden ver, pero no pueden ver la variedad que con frecuencia está alrededor de su cara.

\section{Investigaclón etnográfica}

Iniciando en la mitad de los ochentas, la antropología cultural tuvo una extraordinaria reconsideración de su metodología en un acercamiento etnográfico como su primera forma metodológica, como resultado de las reflexiones metodológicas en las especificidades retóricas de la cultura y las relaciones de poder implícitas en los modos de conocimiento de los otros. Consciente del peligro de etnocentrismo, Hofstede (1980:21) invita al análisis cultural de su propio trabajo. Un efecto crítico que trascienda las nociones existentes de relativismo 
cultural surge a la superficie ilustrando no solamente como la teoría de Hofstede está orientada por su propia cultura sino también construye y sirve al poder y al estatus de su cultura en relación con otras culturas.

Las recientes contribuciones para discutir la investigación etnográfica de los negocios internacionales no cita a importantes fuentes de la teoría postmoderna y postcolonial (Sharpe, 2004). La simplificación de las metáforas para explicar los fenómenos de la administración internacional ha jugado un papel especial en el descenso de los trabajos académicos por la limitada investigación etnocéntrica en donde los prejuicios asociados con un lente focal simple son reconocidos como parroquiales aunque todas las cosas sean recomendadas como universales y juzgadas en relación al enfoque. El etnocentrismo americano es criticado por un relativismo cultural que reconoce las ideas, teorías y prácticas. El intento de quebrar con el etnocentrismo e introducir un recuento científico que trascienda la narrativa occidental tiene límites.

\section{Conclusiones}

En este trabajo se demuestra que la investigación de la administración internacional ha evolucionado en concordancia con la evolución de las concepciones epistemológicas que sustentan los fundamentos teóricos metodológicos de la ciencia. Estas concepciones establecen su dominio como paradigmas de las explicaciones científicas de la realidad de los fenómenos y alcanzan su madurez en la misma época en que tienen una mayor influencia en las actividades de investigación de las ciencias administrativas en el ámbito internacional.

Se demuestra en este trabajo que la construcción teórico metodológica de explicaciones de la realidad de los fenómenos de la administración internacional de las organizaciones ha evolucionado en un continuo pendular sujeto a los paradigmas de la ciencia que prevalecen en la misma época y que se expresan bajo formatos de positivismo funcional en un extremo hasta la investigación cualitativa y etnográfica en el otro extremo, cada uno con sus propios alcances y limitaciones.

Quienes se aferran al uso de un enfoque o corriente teórica determinada limitan los alcances para comprender la compleja realidad de los fenómenos de la administración internacional de las organizaciones y para propiciar la aplicación de principios para la transformación de esta realidad. 


\section{Referenclas}

ALVAREZ, J., MAZZA, C. PEDERSEN, J., Svejenova, S. Shielding idiosyncrasy from isomorphic pressures: towards optimal distinctiveness in European filmmaking. Organization, vol 12, n 6, pp. 863-888, 2005.

ALVESSON, M., Deetz, S. Critical theory and postmodernism: approaches to organizational studies. In S. Clegg S., HARDY, C. (Eds.) Studying organizations: theory and method. London: Sage, 1999, pp. 185-211.

BARLEY, S. R. And Tolbert, P.S. Institutionalization and structuration: Studying the links between action and institution. Organization Science, vol. 18:, p 03-117, 1997.

BARNEY, J. Firm resource and sustainable competitive advantage. Journal of Management, vol.17, n 1, p. 99-120, 1991.

BARNEY, J. B.; ARIKAN, A. M. The resource-based view: origins and implications In: HITT, M. A.; FREEMAN, R.E.; HARRISON, J.S. (Eds.).The Blackwell Handbook of Strategic Management. Oxford:Blackwell, 2001.

BARTLET, C. A., GHOSAL, B and BIRKINSHAW, J, M. Transnational management: text, cases, and readings in cross border management ( ${ }^{\mathrm{a}}$ Ed.). Boston: Harvard Business school press, 2004.

BOEKLE. Henning; RITTBERGER, Volver., WAGNER, Wolfgang (2001). Constructivist foreign policy theory. In: German foreign policy since unification: theories and case studies. Manchester: Manchester University Press, 2001.

BRAITHWAITH, J. And DRAHOS, P. Global business regulation. New York: Cambridge University Press, 2000.

BURGOS SILVA, Germán. "Estado de Derecho y desarrollo económico: aportes y limitaciones de los análisis neinstitucionales". Revista Instituciones y Desarrollo, no. 12-13, p.193-221. Institut Internacional de Governabilitat de Catalunya, Barcelona. 2002.

CHECKEL, Jeffrey. The constructivist turn in IR Theory. World Politics, vol. 50, p.324-348, 1998.

CLEMENS, E. S. and COOK, J. M. Politics and Institutionalism: explaining durability and change. Annual review of sociology, vol. 26, p.441-466, 1999 .

COMMONS, J. R. The economics of collective action. Madison: University of

Wisconsin Press, 1950.

DEETZ, S. Democracy in an age of corporate colonization: Development in communication and the politics of everyday life. Albany: State University of New York, 1992.

DEETZ, S. Critical-interpretative research in organizational communication. Western Journal of Speech, vol. 46, p.131-149, 1982.

DIMAGGIO, P.J.; POWELL, W.W. Introduction. In: POWELL, W.W.; DIMAGGIO,

P.J. (org). The New Institutionalism in Organizational Analysis. Chicago and

London: The University of Chicago Press, 1991. p. 1-38.

DIMAGGIO, P. J. and POWELL, W.W. The iron cage revisited: institutional isomorphism and collective rationality in organizational fields. American Sociological Review, vol. 48, n² 2, p.147-160, 1983.

ESTEFANÍA, Joaquín. "La enfermedad moral del capitalismo". Granito de arena. 7 de Agosto de 2002. Argentina.

EVANS, P. Dependent development: The alliance of multinational, state, and local capital in Brazil. Princeton, NJ: Princeton University Press, 1979.

FINNEMORE, M; SIKKINK, K. Taking stock: the constructivist research program in International Relations and comparative politics. Annual Reviews in Political Sciences, 4, 2001, p. 391-416.

FINNEMORE, Marta and SIKKINK, Kathryn. Internacional norm dynamics and political change. International Organization, vol. 52, p. $887-917,1998$. 
Contínuo pendular de la Investigación en la administración

internacional de las organizaciones: del positivismo funcionalista

a la Investlgaclón cualltatlva y etnográflca.

FLIGSTEIN, N. Social skill and institutional theory. American behavioral scientist, vol 40, p.397-405, 1997.

FOUCAULT, M. Language, counter-memory, practice: selected essays and interviews. Oxford: Blackwell, 1979.

GADAMER,H. G. Truth and method. New York: Crossroad, 1975.

GEPHART, R. P. Qualitative research and the Academy of Management Journal. Academy of Management Journal, vol 47, p.454462, 2004 .

GREENWOOD, R. and HININGS, C. R. Understanding radical organizational change: Bringing together the old and the new institutionalism, Academy of Management Review, vol. 21, p.1022-1105, 1996.

HABERMAS, J. The theory of communicative action: reason and the rationalization of society. Boston: Beacon Press, 1984.

HARDY, C. and PHILLIPS, N. Strategies of engagement: lessons from the critica examination of collaboration and conflict in an organizational domain. Organization Science, vol 9, p. 217-230, 1998.

HIRSCH, P.; LOUNSBURY, M. Ending the family quarrel: toward a reconciliation of "old" and "new" institutionalism. The American Behavioral Scientist, n. 40, v. 4, p. 406-418, Feb. 1997.

HUSSERL, E. Cartesian Meditations: An Introduction to Phenomenology. Nijhoff: The Hague, 1960.

HOFFMAN, A. Institutional evolution and change: enviromentalism and the U.S.

Chemical Industry. Academy of Management Jounal, v.42,n.4, p.351-371, ago/1999.

HOFSTEDE, G. An American in Paris: the influence of nationality on organization theories. Organization Studies, V. 17, n. 3, p. 525$537,1996$.

HOFSTEDE, G. Management scientists are human. Management Science, v. 40, n.1, p. 4,-. 10, 1994

HOFSTEDE, G. Cultural constraints in management theories. Academy of Management Executive, vol. 7, no 1, p.81-94, 1993.

HOFSTEDE, G. The cultural relativity of organizational practices and theories. Journal of International Business Studies, vol.14, $\mathrm{n}^{\circ} 2$, p.75-89, 1983 .

HOFSTEDE, G. Motivation, leadership, and organization: do American theories

applied abroad? Organizational Dynamics, vol. 9, vol.1, p.42-63, 1980a.

HOFSTEDE, G. Culture's consequences: internacional diferencies in work-related values. Beverly Hills, CA: Sage, 1980b.

JACK, G., WESTWOOD, R. Postcolonialism and the politics of qualitative research in international business. Management International Review, vol. 46, p.481-501, 2006.

KIM, B., PRESCOTT, J. E., KIM, S. M. Differentiated governance of foreign subsidiaries in transnational corporations: an agency theory perspectivas. Journal of International Management, vol.11, p.43-66, 2005.

KOSTOVA, T. Transnational transfer of strategic organizational practices contextual perspective. Academy of Management Review, v. 24, n. 2, p. 308-324, 1999.

KOSTOVA, T., ROTH, K. Adoption of an organizational practice by subsidiaries of multinational corporations: Institutional and relational effects. Academy of Management Journal, vol. 45, nº 1, p.215-243, 2002.

KOSTOVA, T. and ZAHEER, S. Organizational legitimacy under conditions of complexity: The case of the multinational enterprise. Academy of Management Review, vol. 24, p.64-81, 1999.

KOSTOVA, T., DACIN, M. Theorizing on MNCs: a promise for institutional theory. Academy of Management Review, vol. 34, $\mathrm{n}^{\circ} 1, \mathrm{p}$. 171-173, 2008. 
KOSTOVA, T. Roth., K. AND DACIN, M. Institutional theory in the study of multinational corporations: a critique and new directions. Academy of Management Review, vol. 33, nº. 4, p.994-1006, 2008.

LAWRENCE, T., PHILLIPS, N. From Moby Dick to Free Willy. Macro cultural discourse and institutional entrepreneurship in emerging institutional fields. Organization, vol.11, p.689-711, 2004.

LEONARDI, Paul M. Indeterminacy and the discourse of inevitability in international technology management. Academy of Management Review, vol. 33, nº 4, p.975-984, 2008.

LOUNSBURY, M.,VENTRESCA, M. The new structuralism in organizational theory. Organization, vol.10, p.457-480, 2003.

MCADAM, D., SCOTT, W.R. Organizations and movements. In: DAVIS, G. F. et al. (Eds.). Social movements and organization theory. Cambridge: Cambridge University Press, 2005.

MEYER, J.; ROWAN, B. Institutionalized organizations: formal structure as myth and ceremony. American Journal of Sociology, v. 83, n. 2, p. 340-363, 1977 .

MILLER, Clark., PAUL N. Edwards. Changing the atmosphere: Expert knowledge and environmental governance. Cambridge MA: MIT Press, 2001.

NORTH, D.D. Institutions, Institutional Change and Economic Performance, Cambridge: Cambridge University Press, 1991.

NORTH, D.D. Institutions, institutional change and performance. New York:

Cambridge University Press, 1990.

OLIVER, C. Strategic responses to institutional processes. Academy of Management Review, Vol.16, p.145-179, 1991.

PELTONEN, T. Critical theoretical perspectives on international human resource management. In: STAHL, G. K., BJORKMAN, J. (Eds.). Handbook of Research in International Human Resource Management:, p.523-535. Cheltenham UK: Edward Elgar, 2006.

PHILLIPS, N., TRACEY, P. Dialogue. Academy of Management Review, vol 34, nº11, p.169-173, 2009.

PORTER, M. E. Competitive advantage: Creating and sustaining superior performance. New York: Free Press, 1985.

RAO, H., Morril, C., Zald, M.N. Power plays: how social movements and collective action create new organizational forms. Research in Organizational Behavior, vol. 22, p.239-282, 2000.

SCOTT, W. R. Institutions and organizations. Thousand Oaks, Ca: Sage, 2001.

SCOTT, W. H. Institutions and organizations. Thousand Oaks CA: Sage, 1995.

SHARPE, D. R. The relevance of ethnography to international business research. In: B. Marschan-Piekkari and C. Welch (Eds.) Handbook of qualitative research: methods for international business, p.306-323. Cheltenham, UK: Edward Elgar, 2004.

SIRMON, D. G., GOVE, STEVE., HITT, M. A. Resource management in dyadic competitive rivalry: the effects of resource bundling and deployment. Academy of Management Journal, vol. 51, nº. 5, p.919-935, 2008.

STEELE, Brent J. Liberal-idealismo: a constructivist critique. International Studies Review, vol. 9, p.23-52, 2007.

SUDDABY, R., GREENWOOD, R. Rethorical strategies of legitimacy. Administrative Science Quarterly, 50: 35-57, 2005.

TEECE, D. J., PISANO, G., SHUEN, A. Dinamic capabilities and strategic management. Strategic Management Journal, vol.18, p.509-533, 1997.

VAARA, E., TIENARI, J., LAURILA, J. Pulp and paper fiction: on the discursive legitimation of global industrial reestructuring", Organization Studies, 27, p.789-310, 2006.

WAJAMAN, J. Feminist theories of technology. In: JASANOFF, S.; MARKLE, G.E.; PETERSON, J. E.; PINCH, T. (Eds.) The handbook of science and technology Studies, p.189-204. Thousand Oaaks, CA: Sage, 1995. 
WALLERSTEIN, I. World-system analysis: an introduction. Durham, NC: Duke University Press, 2004.

WESTPHAL, James D., CLEMENT, Michael B. Sociopolitical dynamics in relations between top managers and security analysts: favor rendering, reciprocity, and analyst stock recommendations. Academy of Management Journal, vol. 51, nº. 5, p.873-897, 2008.

WESTWOOD, R Towards a postcolonial research paradigm in international business and comparative management. In: MARSCHANPIEKKARI, R., WELCH, C. (Eds.). Handbook of qualitative research methods for international business, p.56-83. Cheltenham, UK: Edward Elgar, 2004.

WILLIAMSON, O. E. The mechanisms of governance., New York: Oxford University Press, 1996.

WILLIAMSON, O. E. Transaction cost economics: the governance of contractual relations. Journal of Law and economics, vol. 22:, p.233-262, 1979 .

WILLIAMSON, O. E. Markets and hierarchies; analysis and antitrust implications. New York: Free Press, 1975.

Zacher, Mark., Sutton, Brent. Governing global networks: international regimes for transportation and communications. Cambridge: Cambridge University Press, 1998. 